Session 2432

\title{
The INFINITY Project: Building a High School Curriculum Focused on Modern Technology Which Emphasizes Engineering, Math, and Science Principles
}

\author{
Mark A. Yoder ${ }^{1}$, Ravi Athale ${ }^{2}$, Scott Douglas $^{3}$, Dave Munson $^{4}$, \\ Geoffrey Orsak ${ }^{3}$, John Treichler ${ }^{5}$, Sally Wood ${ }^{6}$ \\ ${ }^{1}$ Rose-Hulman Institute of Technology / ${ }^{2}$ George Mason University / \\ ${ }^{3}$ Southern Methodist University / ${ }^{4}$ University of Illinois / \\ ${ }^{5}$ Applied Signal Technology / ${ }^{6}$ Santa Clara University
}

\begin{abstract}
The INFINITY Project (www.infinity-project.org) is a nationally recognized partnership between leading universities, industry, and K-12 educators to help school districts incorporate modern engineering and technology in their high school curricula. This effort aims to develop a novel and interactive curriculum emphasizing the engineering fundamentals and the fun of the modern hightech age.
\end{abstract}

This paper outlines the goals and technology elements of the INFINITY Project. Implementation issues of the program are addressed, including textbook and laboratory creation, teacher training, and online classroom support.

Math and physics high school teachers are teaching this curriculum in 14 Texas schools at this time. This paper presents the early results of this new course for high school juniors or seniors. Initial responses to the effort from teacher and students have been positive.

\section{Introduction}

Engineers make up a vital part of today's high-tech world. The Internet, PDA's, cell phones, video games, and other fruits of engineering labor are used by millions of people in their daily lives. In highly developed countries, many children are exposed to advanced technology at a young age through computers, video games, digital audio and video, email, etc. Because of 
modern-day pre-college curricula, however, many of these children will never learn about the mathematical and scientific underpinnings of our society's ubiquitous high technology. What skills will prepare students for the challenges of a high-tech society? Clearly, basic knowledge of both mathematics and science are critical. It is our belief, however, that an understanding of how engineers use math and science in technology development is equally crucial to students' success. The best way to ensure that students gain that knowledge is to include engineering and technology as part of the core subjects in elementary and high school educations. The INFINITY Project presents our combined efforts towards this goal.

This paper outlines the goals and technology elements of the INFINITY Project, a joint effort between university educators, K-12 teachers, and industrial partners to introduce an engineering and technology curriculum at the high school level. The partner institute and companies are: Southern Methodist University, Rose-Hulman Institute of Technology, George Mason University, University of Illinois, Santa Clara University, Texas Instruments, Hyperception, Inc., Applied Signal Technology, the National Science Foundation, and regional school districts across the country.

Issues addressed within this paper are:

- The goals and description of the curriculum;

- Technology used in the curriculum;

- Teacher training;

- The pilot program; and

- Future directions.

It is our hope that these issues will serve as a model for the implementation of similar efforts in other educational fields and in engineering education as a whole.

\section{Curriculum Goals}

The curriculum for the INFINITY Project has been designed to meet certain goals in terms of subject areas, scope, and difficulty level. These goals are:

1. The curriculum must be teachable by existing high school educators drawn from the mathematics or science fields. This is motivated by practical concerns; schools can't be expected to hire engineers to teach a new class. Also, the new curriculum needs to fit with the present math and science structure, rather than replace it. Such integration is best served by employing the teachers already in place at the high schools.

2. The subject areas must include topics that are practical and relevant to the students as a whole. The students are already exposed to many high-tech things (compact disc (CD), digital versatile disc (DVD), MPEG I Audio Layer 3 (MP3), etc.), so why not build a curriculum around 
the things students have already seen? Our curriculum emphasis is not focused on the practical details of technologies that are likely to change. Instead, an emphasis is placed on the long-term issues of engineering design and fundamental mathematical and scientific concepts that are the underpinnings of both existing and future technological developments.

3. Hands-on experiments, along with supporting hardware and software. Going hands-on is a must for making the subject "come alive" for the students. We have partnered with Texas Instruments and Hyperception, Inc. to develop the INFINITY Technology Kit to meet these needs. This kit will be covered in more detail later.

4. The presentation must encourage even non-technical students to understand what's behind technology. Technology is everywhere, therefore even budding doctors and lawyers should have some idea what it is.

\section{Curriculum Description}

As in all educational pursuits, the INFINITY curriculum requires a core discipline upon which to build fundamental concepts and pedagogy. We believe that the field of multimedia-based signal and information processing, as manifested in wireless communications, computer networks, and digital entertainment, represents an ideal core subject for teaching engineering fundamentals. The level of recreational interest high school students show in these topics support our choice. We also believe that these topics offer the right balance of basic mathematics and science for the proposed educational experience.

We have written a textbook embodies the curriculum's goal and focus [1]. Consisting of over 600 pages of text and 500 figures organized into twenty chapters, the textbook covers nearly all aspects of modern-day multimedia and information technology. The main topics include:

- Definitions and descriptions of digital and analog technology;

- The engineering design process;

- Fundamental systems concepts (input/output behavior, frequency response)

- The basics of hearing and sight perception relevant to digital audio and imaging;

- The physics and mathematics of electronic and optical displays;

- Information storage, compression, and encryption;

- Radios and wireless communications; and

- Computer networks and the Internet.

In addition, each chapter contains review questions designed to test comprehension and mathematical understanding of main concepts. 


\section{Technology}

Along with the textbook, we have designed laboratory experiments that employ the INFINITY Technology Kit to teach important engineering concepts using real-time DSP (digital signal processing) hardware and software. These experiments are best described as "digital worksheets" within the Hyperception (http://www.hypercpetion.com) block diagram software environment, in which students can explore existing and create new real-time signal processing systems through a point-and-click user interface. Examples of some of the experiments include:

- A digital imaging based coin counter that determines the number of a certain type of coin in a real-time video signal stream;

- A MIDI musical instrument synthesizer;

- A real-time digital transmitter and receiver for sending text messages across the room using loudspeakers and microphones;

- A real-time object tracker (shown in Figure 1); and

- A sock matched to help a blind person select socks.

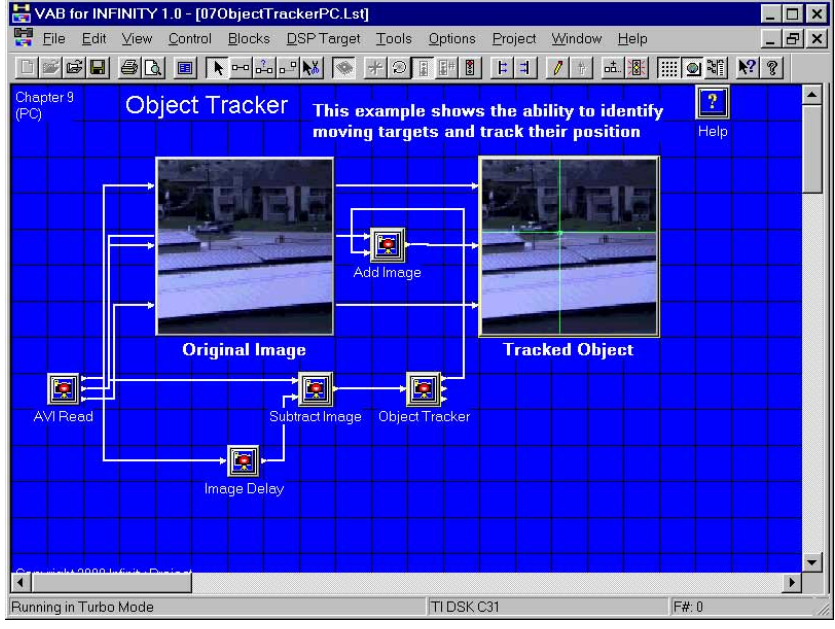

Figure 1. A VAB worksheet.

The INFINITY Technology Kit includes (see Figure 2):

- DSP Hardware:

Texas Instrument's TMS320C31 DSP starter kit.

- DSP Software: Hyperception's Visual Application Builder.

- Multimedia Accessories: $\quad$ Powered speakers, microphone with preamplifier, audio adapter, audio cable, AC power supply, stereo glasses (red/blue), 9V battery.

The kit designed to be a complete and robust system for students to use in a typical high school laboratory setting. It easily installs on a standard PC. In addition, a low-cost Internet video camera is suggested but not required for image and video experiments. 


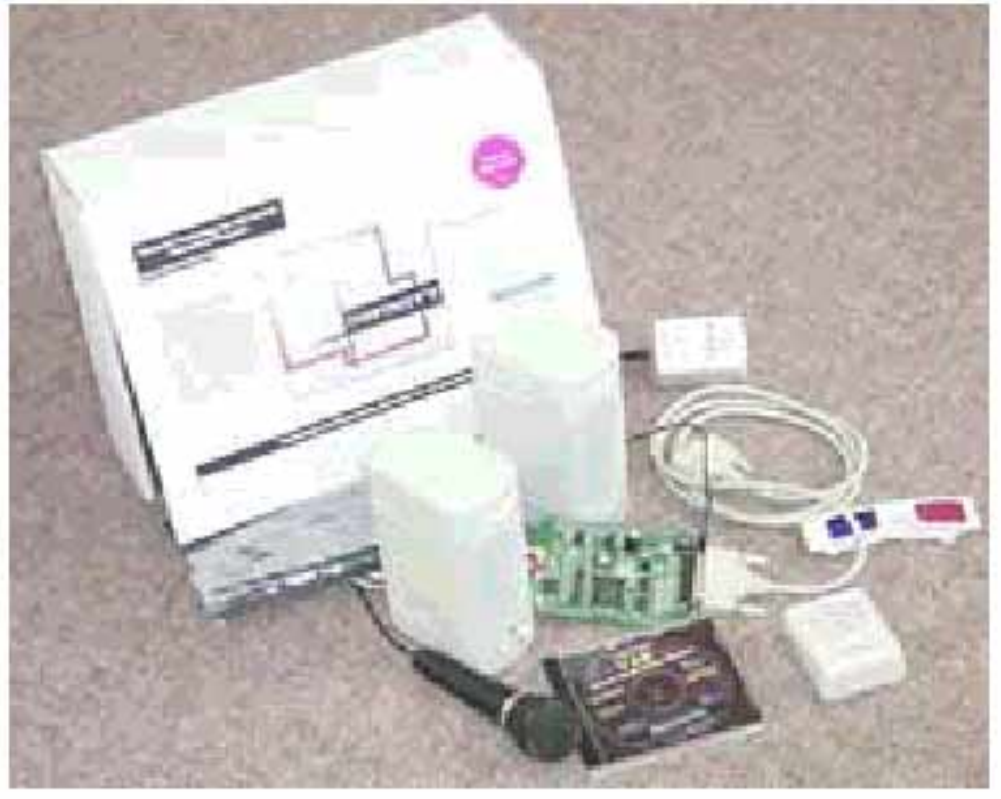

Figure 2. The INFINITY Technology Kit.

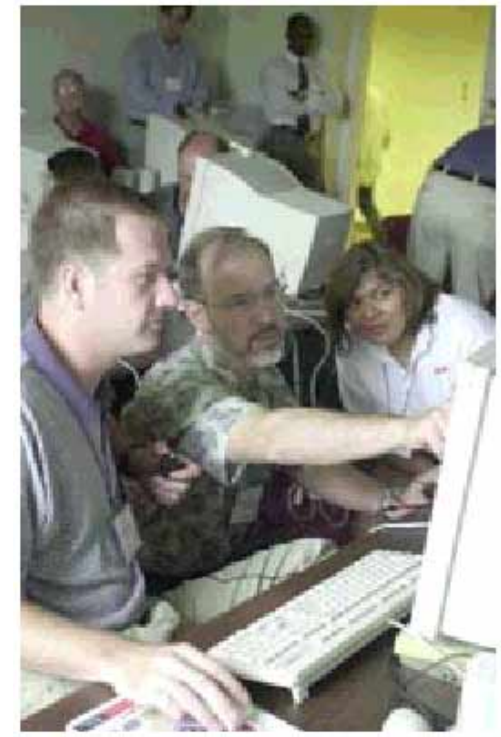

Figure 3. The Infinity Institute.

\section{Teacher training}

Teacher training is a major component of the INFINITY Project. We understand that to effectively teach engineering and advanced technology at the high school level, teacher must be supplied with more than an innovative curriculum and modern equipment. Teachers must also have access to training that directly helps them teach the material and operate the equipment in a classroom setting.

To fill this important need, the INFINITY Project currently trains all teacher of the new engineering curriculum by experts in the curriculum. This training covers the basics of the course material as well as those of the INFINITY Technology Kit. Our INFINITY training institute is 40 hours long and is usually conducted over a one-week period. Onr institute has been designed to be "hands-on" for the high school teachers. Teachers new to technology are given many opportunities to work with the equipment and apply the new material to real problems in a supportive and fun environment.

During the summer of 2000, we offered two training Institutes that trained 25 high school teachers on all aspects of the INFINITY curriculum. The responses of the teachers was entirely positive and enthusiastic, as measured from evaluation given to every teacher at the end of the training period. Figure 3 is a picture from the training. 
VI. Implementation and Results

In the current 2000-2001 inaugural year of the INFINITY Project, our high-technology curriculum is being taught to approximately 500 students in fourteen different high schools in the Dallas, Houston, and San Antonio, TX areas. These school include both public and private institutions of single-gender and mixed-gender students at urban and suburban campuses. Figure 4 shows a typical laboratory session at one of the high schools.

\section{Future Directions}

As for expansion of the program, our current plans have the Infinity curriculum being offered at 40 different high schools in the 2001-2002 academic year, with 30 of these in Texas and the remaining 10 in other state across the United States. Interest in the program has exceeded our capabilities to meet the demand;

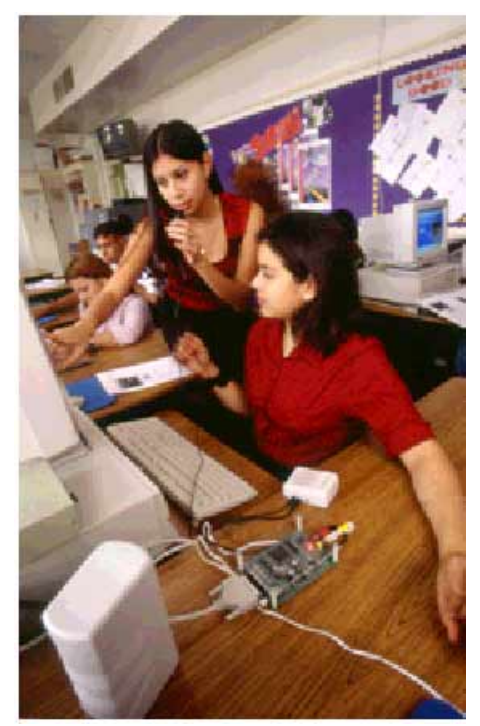

Figure 4. INFINITY students in the high school classroom. there are many more schools that wish to offer this program. To meet this demand, we intend to adopt a growth method similar to that used by the Teacher Teaching with Technology $\left(\mathrm{T}^{3}\right)$ program for introducing electronic calculators into high school math classes (http://www.t3ww.org). In that model, select teachers who have been trained become certified trainers themselves, thereby allowing exponential growth of trainers to meet an exponential growth in training demand. From our current projection for the program, we expect to educate a total of 100,000 students in the INFINITY Project curriculum by 2005.

\section{Conclusions}

The Infinity Project is a curriculum development and implementation effort designed to introduce high school students to the critical thinking, principles, and excitement of engineering. This paper described the initial efforts on this two-year-old project. Further developments, as well as additional details regarding the goals, implementation, and progress of the Infinity Project, can be found at the Project web page (http://www.infinity-project.org)

\section{References}

[1] R. A. Athale, S. C Douglas, D. C. Munson, Jr., G.C. Orsak, J. R. Treichler, S. L Wood, and M. A. Yoder, Multimedia and Information Engineering, textbook draft, to be published. 


\section{Biography}

MARK A. YODER

Mark is an Associate Professor of Electrical and Computer Engineering at Rose-Hulman Institute of Technology in Terre Haute, Indiana. He co-authored the book DSP First: A Multimedia Approach with Jim McClellan and Ron Schafer. Mark's biography isn't complete without some mention of his family. His wife Sarah has her Ph.D. in Electrical Engineering from Purdue University, and they have nine wonderful children ages 17, 17, 14, 12, 10, 8, 6, 4, and 1 . Three boys and six girls.

\section{RAVi ATHALE}

Dr. Athale is an Associate Professor in the Electrical and Computer Engineering Department at George Mason University, in Fairfax, VA. Dr. Athale was elected Fellow of the Optical Society of America in 1989. Dr. Athale has been issued several patent in optical processing and computing. He is a cofounder of HoloSpexTM, Inc. and a COinventor of HoloSpexTM glasses, the first consumer product that is based on far field holograms.

\section{ScOTT C. DOUGLAS}

Scott C. Douglas is with the Department of Electrical Engineering in the School of Engineering and Applied Science at Southern Methodist University, Dallas, TX, as an Associate Professor. His research activities include adaptive filtering, active noise control, blind deconvolution and source separation, and VLSI/hardware implementations of digital signal processing systems.

DAVID C. MUNSON, JR.

David C. Munson, Jr. is with the University of Illinois at Urbana-Champaign, where he is currently a Professor in the Department of Electrical and Computer Engineering, a Research Professor in the Coordinated Science Laboratory, and a Research Professor in the Beckman Institute for Advanced Science and Technology. His research interests are in the general area of signal and image processing with current work focused on radar imaging, tomography, interferometry, interpolation, time-frequency analysis, and digital filtering.

\section{GEOFFREY C. ORSAK}

Geoffrey C. Orsak is currently an Associate Professor of Electrical Engineering in the School of Engineering and Applied Science at Southern Methodist University . In addition to his departmental responsibilities, he is Director of The INFINITY Project. His research interests are in the area of communication theory, detection and estimation theory, information theory, and statistical signal processing. In addition to this work, he has also been active in the use of high technology for novel forms of pedagogy.

\section{JOHN R. TREICHLER}

In 1984 Dr. Treichler co-founded Applied Signal Technology, Inc. of Sunnyvale, CA in 1984 with three collegues. He is currently the company's Chief Technology Officer and also serves on the company's board of directors. The company designs and builds advanced signal processing equipment which is used by the United States government and its allies. Dr. Treichler was named a Fellow of the IEEE in 1991.

\section{SALLY L. WOOD}

Sally L. Wood joined the faculty of Santa Clara University in 1985 and is currently Professor and Chair of the Electrical Engineering Department. At Santa Clara University Professor Wood has developed and taught courses in signal and image processing at both the undergraduate and graduate level. In addition, she has developed and taught a freshman level laboratory based introductory electrical engineering course and a sophomore level signal processing architecture course.. Her current research interests include multiple source image analysis and nonlinear signal processing.

\section{Proceedings of the 2001 American Society for Engineering Education Annual Conference \& Exposition} Copyright (O) 2001, American Society for Engineering Education 